\title{
Apolipoprotein A-I Mimetic Peptides
}

\author{
Brian J. Van Lenten, PhD, Alan C. Wagner, BS, G. M. Anantharamaiah, PhD, Mohamad \\ Navab, PhD, Srinivasa T. Reddy, PhD, Georgette M. Buga, PhD, and Alan M. Fogelman, MD
}

\section{Abstract}

Recent publications reveal the mechanism of action of apolipoprotein A-I (apoA-I) mimetic peptides to be the remarkable binding affinity that oxidized lipids have for these peptides compared with apoA-I. There was no difference in the binding affinity of oxidized lipids or in peptide efficacy in reducing inflammation and atherosclerosis in rabbits injected with peptides synthesized from all D- or all L-amino acids. The apoA-I mimetic peptide $4 \mathrm{~F}$ increased the formation of pre- $\beta$ high-density lipoprotein, increased cholesterol efflux, and reduced lipoprotein oxidation in vitro; it increased antioxidants and vascular repair in type I diabetic rats; it improved vasodilation, oxidative stress, myocardial inflammation, and angiogenic potential in a mouse model of scleroderma; it reduced renal inflammation in low-density lipoprotein receptor-null mice fed a Western diet; it reduced arthritis in a rat model; it reduced adiposity, increased adiponectin levels, and improved insulin sensitivity in obese mice; and it improved high-density lipoprotein inflammatory properties in humans with coronary heart disease.

\section{Introduction}

There is a growing awareness that high-density lipoprotein (HDL) cholesterol levels are not sufficient for predicting HDL function [1]. The beneficial effects of HDL have largely been attributed to its major protein, apolipoprotein A-I (apoA-I). Decades of research have gone into the synthesis of apoA-I mimetic peptides. Initial efforts concentrated on synthesizing peptides that, similar to apoA-I, bound non-oxidized lipids and activated lecithincholesterol acyl transferase (LCAT), similar to apoA-I. However, an 18-amino acid peptide that bound non-oxidized lipids similarly to apoA-I and activated LCAT was not effective in mouse models of atherosclerosis [2]. Instead of screening for non-oxidized lipid binding and LCAT activation, an in vitro model of the human artery wall was used to screen potential apoA-I mimetic peptides for their ability to inhibit low-density lipoprotein (LDL) oxidation and LDL-induced production of the potent monocyte chemoattractant protein-1 (MCP-1). This new approach led to the identification of peptides that demonstrated anti-inflammatory and anti-atheroscleroti c activity in animal models [3]. This review focuses on recent publications regarding this emerging class of therapeutic agents.

Copyright $\odot 2009$ by Current Medicine Group LLC

Corresponding author Brian J. Van Lenten, PhD, Room BH-307 CHS, Division of Cardiology, Department of Medicine, David Geffen School of Medicine at UCLA, 10833 Le Conte Avenue, Los Angeles, CA 90095, USA. bvanlent@mednet.ucla.edu.

Disclosures

Dr. Navab, Dr. Anantharamaiah, and Dr. Fogelman are principals in Bruin Pharma, and Dr. Fogelman is an officer in Bruin Pharma. No other potential conflicts of interest relevant to this article were reported. 


\section{Review of Recent Literature}

\section{Structure of apoA-I mimetic peptides}

The structural requirements for the antioxidative and anti-inflammatory properties of apoA-I mimetic peptides have recently been described [4•]. As noted previously, peptides mimicking apoA-I that are anti-atherogenic and anti-inflammatory bind non-oxidized lipids and activate LCAT similarly to peptides that are not antiatherogenic or anti-inflammatory. Using physical-chemical and nuclear magnetic resonance studies, it was found that peptides that interact strongly with lipid acyl chains do not have anti-atherogenic and antiinflammatory properties, but peptides that associate closely to the lipid head group (and hence do not strongly interact with the lipid acyl chain) are anti-atherogenic and antiinflammatory. The difference was postulated to be in the microenvironment created by these peptides, which did or did not favor the sequestration of pro-inflammatory oxidized lipids. Peptides that associate close to the lipid head group (and hence do not strongly interact with the lipid acyl chain) were postulated to provide a microenvironment favoring the sequestration of proinflammatory lipids. In contrast, peptides that interact strongly with lipid acyl chains were postulated not to provide a microenvironment favorable to the sequestration of pro-inflammatory lipids $[4 \bullet]$.

\section{Mechanism of action of antiatherogenic and anti-inflammatory apoA-I mimetic peptides}

Among the best studied apoA-I mimetic peptides are the 4F peptides, which have been synthesized from both all D-amino acids (D-4F) and all L-amino acids (L-4F). The concentration of apoA-I (a $28-\mathrm{kDa}$ protein) in human and mouse plasma is approximately 35 $\mu \mathrm{M}$. The $4 \mathrm{~F}$ peptides have a molecular weight of $2.3 \mathrm{kDa}$. After oral administration of $\mathrm{D}-4 \mathrm{~F}$ to mice, the maximal plasma concentration achieved was only approximately $130 \mathrm{nM}$ [5]. As will be discussed in more detail in the following text, humans who received a single oral dose of D-4F had maximal plasma concentrations of only approximately $4 \mathrm{nM}[6 \bullet]$. How could $4-130 \mathrm{nM}$ of an apoA-I mimetic peptide be biologically active in the presence of 35 $\mu \mathrm{M}$ of apoA-I?

The answer to this conundrum was suggested by the finding that adding human apoA-I to human artery wall cells in a preincubation followed by removal of the apoA-I prior to the addition of human LDL dramatically reduced the ability of the cells to oxidize LDL to a form that could stimulate the induction of MCP-1 as determined by a bioassay [7]. However, if the apoA-I was left in the cultures when the LDL was added (a coincubation), the oxidation of LDL and the induction of MCP-1 was the same as if the apoA-I had not been added [7]. In contrast, the addition of 4F was effective even in a coincubation [2]. This led to the hypothesis that apoA-I bound the proinflammatory oxidized lipids generated by the interaction of the human artery wall cells with LDL with lower affinity compared with $4 \mathrm{~F}$, allowing the lipids to dissociate from apoA-I and stimulate the cells to produce MCP-1. In contrast, it was hypothesized that the affinity of the oxidized lipids for $4 \mathrm{~F}$ was so strong that they could not effectively dissociate and MCP-1 production was prevented [3].

Van Lenten et al. [8••] directly tested and confirmed this hypothesis. They used surface plasmon resonance measurements to determine the binding affinities of both non-oxidized and oxidized lipids for D-4F and L-4F compared with full-length human apoA-I. They found no significant differences between D-4F and L-4F and they found no significant difference in the abilities of the $4 \mathrm{~F}$ peptides and apoA-I to bind non-oxidized phospholipids. However, they found that the 4F peptides bound oxidized phospholipids with much higher affinity than the parent non-oxidized lipids, but that this was not the case for apoA-I. The 4F peptides and apoA-I bound non-oxidized fatty acids with equally high affinity. However, 
when the fatty acids were oxidized apoA-I no longer bound them with high affinity, whereas the $4 \mathrm{~F}$ peptides did.

Binding affinity is measured by a constant called $\mathrm{K}_{\mathrm{D}}$, where $\mathrm{K}_{\mathrm{D}}$ is equal to $\mathrm{Kd}$ (the dissociation rate constant) divided by $\mathrm{Ka}$ (the association rate constant). The larger the $\mathrm{K}_{\mathrm{D}}$ value, the weaker is the binding. Conversely, the smaller the $\mathrm{K}_{\mathrm{D}}$ value, the stronger is the binding. Van Lenten et al. [8••] found that the KD for the binding of a non-oxidized, noninflammatory phospholipid found in LDL (PAPC) was 118,576 $\pm 36,843,192,821 \pm 56,505$, and 99,871 $\pm 14,114 \mathrm{nM}$ for D-4F, L-4F, and human apoA-I, respectively. PEIPC is an isoprostane-like oxidized phospholipid that is very pro-inflammatory and derived from the oxidation of the parent non-oxidized, non-inflammatory phospholipid PAPC. The $\mathrm{K}_{\mathrm{D}}$ for the binding of PEIPC was $0.06 \pm 0.05,0.01 \pm 0.01$, and 50,720 $\pm 5,721 \mathrm{nM}$ for D-4F, L-4F, and human apoA-I, respectively. Thus, the binding affinity of this pro-inflammatory oxidized phospholipid for the $4 \mathrm{~F}$ peptides was 842,833-fold to 5,072,000-fold greater than for human apoA-I. Van Lenten et al. [8••] also demonstrated that $35 \mu \mathrm{M}$ of human apoA-I added to cultures of human aortic endothelial cells did not inhibit LDL-induced MCP-1 production. However, adding L-4F at a concentration of $4.3 \mathrm{nM}$ in the presence of $35 \mu \mathrm{M}$ of human apoA-I significantly reduced LDL-induced MCP-1 production $(P<0.001)$. Thus, the mechanism of action of these apoA-I mimetic peptides was shown to be due to the remarkable affinity of the pro-inflammatory oxidized lipids for the peptides compared with apoA-I [8••].

\section{Comparison of the anti-inflammatory and anti-atherogenic properties of D-4F versus L-4F}

As noted previously, the binding affinities of non-oxidized and oxidized lipids are the same for D-4F and L-4F. To test the efficacy of these two peptides in vivo, Van Lenten et al. [9•] used a rabbit model. New Zealand white rabbits were fed a chow diet containing $1 \%$ cholesterol for 1 month. After 1 month on the 1\%-cholesterol diet, plasma cholesterol concentrations were measured and groups of three rabbits with similar plasma cholesterol levels were randomly assigned to each of three treatment groups so that the plasma cholesterol concentrations of the three treatment groups were not significantly different. The $1 \%$-cholesterol diet was continued and the rabbits (which weighed about $4 \mathrm{~kg}$ ) were administered $10 \mathrm{mg} / \mathrm{kg}$ per day of D-4F or L-4F either subcutaneously or via vehicle. After 1 month, the rabbits were sacrificed. The percent of aorta with atherosclerotic lesions was $24 \% \pm 15 \%$ (vehicle), $10 \% \pm 6 \%$ (D-4F) $(P<0.01$ versus vehicle), and $13 \% \pm 9 \%(\mathrm{~L}-4 \mathrm{~F})(P$ $<0.05$ versus vehicle). Inflammatory indexes for HDL and LDL were determined by measuring MCP-1 levels by bioassay in cultures of human aortic endothelial cells. The higher the lipoprotein index values, the more MCP-1 production. The HDL and LDL inflammatory indexes, respectively, were $1.39 \pm 0.24 ; 1.35 \pm 0.29$ for vehicle, $0.67 \pm 0.26$; $0.63 \pm 0.38$ for $\mathrm{D}-4 \mathrm{~F}(P<0.001$ compared with vehicle $)$, and $0.67 \pm 0.2 ; 0.68 \pm 0.32$ for $\mathrm{L}-4 \mathrm{~F}$ ( $P<0.01$ compared to vehicle). Serum amyloid A levels were $95 \pm 39 \mu \mathrm{g} / \mathrm{mL}$ for vehicle-treated rabbits, $8 \pm 22 \mu \mathrm{g} / \mathrm{mL}$ for D- $4 \mathrm{~F}$-treated rabbits, and $7 \pm 19 \mu \mathrm{g} / \mathrm{mL}$ for L- $4 \mathrm{~F}-$ treated rabbits $(P<0.001$ for $\mathrm{D}-4 \mathrm{~F}$ and $\mathrm{L}-4 \mathrm{~F}$ compared with vehicle). There was no correlation between lesion area and total plasma cholesterol or HDL cholesterol levels. In contrast, there was a positive correlation with the HDL inflammatory index $(P=0.002)$, the LDL inflammatory index $(P=0.0026)$, and serum amyloid A levels $(P=0.0079)$. The HDL-inflammatory index correlated closely with the serum amyloid A levels $\left(r=0.6616 ; r^{2}\right.$ $=0.4377 ; P<0.0001$ ). It was concluded that there was no difference in efficacy between $\mathrm{D}-4 \mathrm{~F}$ and L-4F when administered by subcutaneous injection and that the lipoprotein inflammatory indexes, and serum amyloid A levels were better predictors of lesion area than were total plasma cholesterol or HDL cholesterol levels in this rabbit model of atherosclerosis [9•]. 


\section{Pre- $\beta_{1}$ HDL formation}

Pre- $\beta_{1}$ HDL is the form of HDL that is most active in promoting cellular cholesterol efflux by the transporter ATP binding cassette A1 (ABCA1). Troutt et al. (10) reported that D-4F dose dependently induced Pre- $\beta_{1}$ HDL formation in vitro in human plasma and in mice. They also reported that minor modification of the sequence caused the loss of this activity.

\section{Comparison of modified four peptides with $4 \mathrm{~F}$}

Wool et al. [11] hypothesized that apoA-I mimetic peptides that better mimicked the punctuated alpha-helical repeats of full-length apoA-I would be more anti-inflammatory and more antiatherogenic. To test this hypothesis they synthesized L-4F (an 18-amino acid peptide), two 4F peptides linked by a proline (a 37-amino acid peptide), two 4F peptides linked by an alanine (a 37-amino acid peptide), and two 4F peptides linked by the interhelical sequence found between helixes 4 and 5 in human apoA-I (a 43-amino acid peptide). All of the peptides cleared turbid lipid suspensions, indicating lipid binding of the non-oxidized lipids. L-4F was most active in this assay. The tandem peptides were more effective in remodeling mouse HDL. All four peptides displaced apoA-I and apoE from the HDL, leaving a larger particle containing apoA-II and the peptide. Despite the loss of the majority of apoA-I from the peptide-remodeled HDL particles, they continued to show ABCG1-mediated cholesterol efflux comparable with apoA-I-containing HDL particles. The tandem peptides showed greater ability to efflux cholesterol from cholesterol-loaded murine macrophages compared with L-4F. L-4F inhibited oxidation of purified mouse HDL, but the alanine tandem peptide increased oxidation. Comparing the tandem 4F-based peptides with L-4F in assays that correlated with suggested anti-inflammatory and antiatherogenic pathways, the tandem 4F-based peptides exceeded L-4F in HDL remodeling and cholesterol efflux but not in protection against LDL oxidation. It was also concluded that these apoA-I mimetic peptides may increase reverse cholesterol transport through both ABCA1 and ABCG1 pathways [11].

\section{$4 \mathrm{~F}$ in a rat model of diabetes}

Peterson et al. [12]• rendered Sprague-Dawley rats diabetic with streptozotocin and administered vehicle or D-4F by intraperitoneal injection at a dose of $1 \mathrm{mg} / \mathrm{kg}$ per day for 6 weeks. D-4F had no significant effect on plasma glucose levels, which were maintained between 240 and $320 \mathrm{mg} / \mathrm{dL}$ in the different groups by using insulin to prevent weight loss. After 6 weeks of D-4F treatment, heme oxygenase-1 activity significantly increased in the heart and aorta by $29 \%$ and $31 \%$ respectively $(P<0.05$ and $P<0.01$, respectively). Circulating endothelial cells were significantly elevated in diabetic rats compared with control rats ( $50 \pm 6$ for diabetic and $4 \pm 3$ endothelial cells $/ \mathrm{mL}$ for control rats). $\mathrm{D}-4 \mathrm{~F}$ treatment did not alter the number of circulating endothelial cells in the control rats but significantly decreased the number of sloughed endothelial cells in the diabetic rats to $20 \pm 3$ cells per milliliter in peripheral blood $(P<0.003$ compared with untreated diabetic rats). The reduction in circulating endothelial cells by $\mathrm{D}-4 \mathrm{~F}$ was accompanied by an increase in intact endothelium as assessed by immunohistochemical staining for $\mathrm{CD} 31+(P<0.05)$. Indeed, $\mathrm{CD} 31+$ staining in the $\mathrm{D}-4 \mathrm{~F}$-treated diabetic rats was not significantly different from the nondiabetic control rats. There was also a reduction in immunoreactive thrombomodulin in the vehicle-treated diabetic rats, and $\mathrm{D}-4 \mathrm{~F}$ treatment returned the thrombomodulin levels to that seen in nondiabetic rats.

The induction of diabetes was accompanied by a decrease in endothelial progenitor cell function. The number of endothelial progenitor cell colonies was $19 \pm 1.3$ colonies in control rats and was reduced to $8.8 \pm 1.3$ colonies in the diabetic rats $(P<0.001)$. In diabetic rats treated with $\mathrm{D}-4 \mathrm{~F}$, the number of endothelial progenitor cell colonies was $17.3 \pm 1.5$ colonies, which was significantly higher than the diabetic rats that were vehicle treated $(P<$ 
0.002), and not significantly different from the control nondiabetic rats. Endothelial progenitor cells cultured from the marrow of diabetic rats treated with vehicle showed reduced levels of heme oxygenase-1 (but not heme oxygenase-2, which is constitutively expressed) and reduced levels of endothelial nitric oxide synthase (eNOS), which were restored by $\mathrm{D}-4 \mathrm{~F}$ treatment to the levels seen in nondiabetic control rats. The serum content of oxidized protein as measured by carbonyl content was elevated in the vehicle-treated diabetic rats compared with the nondiabetic control rats. D-4F treatment significantly attenuated this increase. Oxidized LDL levels increased in the vehicle-treated rats compared with the nondiabetic rats, and D-4F also significantly ameliorated the levels of oxidized LDL [12•].

\section{$4 \mathrm{~F}$ in a mouse model of scleroderma}

Weihrauch et al. [13•] reported on the effects of D-4F in a mouse model of systemic sclerosis (scleroderma). Scleroderma is an autoimmune connective tissue disorder characterized by impaired vascular function, increased oxidative stress, inflammation, and impaired angiogenesis. Tight skin mice with a defect in fibrillin-1 have many of the myocardial and vascular components of humans with scleroderma. These mice were found to have impaired small artery vasodilation mediated by eNOS, which was dramatically improved by treatment with D-4F given by injection at a dose of $1 \mathrm{mg} / \mathrm{kg}$ per day compared with vehicle alone. The tight skin mice and human patients with scleroderma both had proinflammatory HDL as defined by an assay in which HDL susceptibility to copper oxidation is measured in vitro. $\mathrm{D}-4 \mathrm{~F}$ treatment of the mice significantly increased the ability of the mouse HDL to resist copper oxidation without changing HDL cholesterol levels. Angiostatin levels in the plasma of the vehicle-treated tight skin mice and in humans with scleroderma were elevated compared with controls. D-4F treatment of the mice returned the levels to near those seen in control mice. The hearts of the tight skin mice treated with vehicle contained higher levels of angiostatin and autoantibodies against oxidized phospholipids compared with control mice. D-4F treatment significantly decreased myocardial angiostatin levels and reduced the autoantibodies to oxidized phospholipids by more than 50\%. In vitro potential of thin sections of murine hearts was assessed in vitro for vascular endothelial growth factor-induced endothelial cell tube formation. The tight skin mice had impaired angiogenic potential that was significantly improved by D-4F treatment. The authors concluded that D-4F improves vascular function, decreases myocardial inflammation, and restores angiogenic potential in this mouse model of scleroderma. Because human scleroderma patients were found to have proinflammatory HDL and elevated angiostatin levels, the authors speculated that D-4F treatment may be effective in treating the vascular complications in patients with scleroderma [13•].

\section{F in a mouse model of hyperlipidemia-induced renal inflammation}

Buga et al. [14••] studied kidney changes in LDL receptor- null mice fed a Western diet. Mice on the Western diet that received scrambled D-4F (a peptide with the same amino acids as in $\mathrm{D}-4 \mathrm{~F}$ but in an order that renders the peptide ineffective) had increased glomerular cellularity compared with $\mathrm{D}-4 \mathrm{~F}-$ treated mice $(P<0.0001)$. There was also a significantly expanded mesangial area and increased focal adhesion in the glomeruli of the mice on the Western diet treated with scrambled D-4F compared with D-4F $(P<0.001)$. Dilated capillaries were more prevalent in the mice on the Western diet treated with scrambled D-4F compared with D-4F $(P<0.01)$. There was increased MCP-1 in the glomeruli $(P<0.0001)$ and tubulointerstitial areas $(P<0.02)$ in the mice on the Western diet treated with scrambled D-4F compared with D-4F. Consistent with the increased MCP-1 immunostaining, there was increased macrophage content as determined by immunostaining in the mice on the Western diet treated with scrambled D-4F compared with D-4F $(P<$ 0.001). The kidney content of oxidized phospholipids as determined by EO6 
immunostaining significantly increased in mice on the Western diet treated with scrambled D-4F compared with the mice on the chow diet $(P<0.001)$. Treatment with D-4F returned the levels of oxidized phospholipids to that seen in the LDL receptor-null mice on chow. Although there was a dramatic difference in the kidney content of oxidized phospholipids in mice treated with scrambled D-4F compared with $\mathrm{D}-4 \mathrm{~F}$, there was no significant difference in immunostaining for apoB. Placing LDL receptor-null mice on a Western diet dramatically increased the expression of sterol regulatory element-binding protein-1c (SREBP-1c) mRNA $(P<0.001)$. Treatment with D-4F reduced SREBP-1c expression to levels not significantly different from the mice on chow. The levels of SREBP-1c were paralleled by kidney triglyceride levels. There was no significant difference in plasma total cholesterol, HDL cholesterol, triglycerides, blood sugar, or blood pressure in LDL receptornull mice on the Western diet whether they were treated with scrambled D-4F or D-4F. Thus, D-4F treatment specifically and significantly reduced kidney oxidized phospholipid content, SREBP-1c expression, cytokine expression, macrophage infiltration, and histologic changes without altering kidney retention of apoB-containing lipoproteins or changing plasma lipid levels. These data are consistent with the peptide having selectively removed oxidized lipids, which are known to induce MCP-1 expression via a SREBP-mediated pathway $[14 \bullet \cdot]$.

\section{$4 \mathrm{~F}$ in a rat model of arthritis}

Charles-Schoeman et al. [15] studied D-4F in a rat model of collagen-induced arthritis. Rats receiving $10 \mathrm{mg} / \mathrm{kg}$ per day of pravastatin orally together with D-4F by injection at doses of 3 or $12.5 \mathrm{mg} / \mathrm{kg}$ per day had significantly lower clinical arthritis scores than control rats or rats receiving monotherapy or combination therapy at a D-4F dose of $1 \mathrm{mg} / \mathrm{kg}$ per day $(P<$ $0.0001)$. None of the treatments prevented the onset of clinical arthritis. However, by the end of the study on day $28,33 \%$ of the rats receiving combination therapy with a D-4F dose of $12.5 \mathrm{mg} / \mathrm{kg}$ per day and $25 \%$ of rats receiving combination therapy with a D-4F dose of 3 $\mathrm{mg} / \mathrm{kg}$ per day had resolution of clinical arthritis to a score of 1 or less in both hind limbs. In the combination-therapy group in the rats receiving $12.5 \mathrm{mg} / \mathrm{kg}$ per day of D-4F, $17 \%$ of the animals had absolutely no evidence of arthritis on day 28 despite earlier clinical disease. In contrast, all animals in the control group had persistent arthritis scores of 2 or more in at least one hind limb. No evidence of clinical toxicity was seen in any of the treatment groups. Rats given combination therapy with D-4F at doses of $3 \mathrm{mg} / \mathrm{kg}$ per day or $12.5 \mathrm{mg} / \mathrm{kg}$ per day had significantly less erosive disease by radiographs and histology compared with animals administered the noncombination therapies $(P<0.01)$. The combination therapy did not significantly alter the production of antibodies to type II collagen, which was used to induce the arthritis. Rats receiving D-4F and/or pravastatin had significantly lower levels of serum interleukin (IL)-1 $\beta$, IL-18, MCP-1, and tumor necrosis factor- $\alpha$ compared with control animals at the end of the study $(P=0.009-0.02)$. Rats that had good clinical responses to treatment also had statistically significant improvements in their HDL inflammatory index at day 28 compared with baseline $(P<0.000001)$. Conversely, rats that did not respond clinically had no significant difference in the HDL inflammatory index comparing their values at day 0 and at day 28 at the end of the study [15].

\section{$4 \mathrm{~F}$ in a mouse model of diabetes and obesity}

Peterson et al. [16••] hypothesized that $4 \mathrm{~F}$, which induces arterial anti-oxidative enzymes and is vasoprotective in a rat model of diabetes, would ameliorate insulin resistance and diabetes in obese mice. They found that intraperitoneal administration of L-4F by injection at a dose of $2 \mathrm{mg} / \mathrm{kg}$ per day to ob/ob mice for 6 weeks limited weight gain without altering food intake, decreased visceral $(P<0.02)$ and subcutaneous $(P<0.045)$ fat content, decreased plasma IL-1 $\beta$ and IL-6 levels $(P<0.05)$, and increased insulin sensitivity with resulting in decreased serum glucose $(P<0.001)$ and insulin $(P<0.036)$ levels. L-4F 
treatment also increased aortic and bone marrow heme oxygenase- 1 activity and decreased aortic and bone marrow superoxide production $(P<0.001)$. L-4F treatment also increased serum adiponectin levels $(P<0.037)$ and decreased adipogenesis in mouse bone marrow $(P$ $<0.039)$ as well as in cultures of human bone marrow-derived mesenchymal stem cells $(P<$ 0.022). The authors concluded that their data provided evidence for the existence of a temporal relationship between heme oxygenase- 1 and adiponectin that is positively affected by L-4F in ob/ob mice resulting in reduced adiposity, improved insulin resistance, improved glucose tolerance, increased adiponectin levels, and reduced IL-1 $\beta$ and IL-6 levels in this mouse model of diabetes and obesity [16••].

\section{F improves HDL inflammatory properties in humans with coronary heart disease}

The first test of $4 \mathrm{~F}$ in humans was recently reported by Bloedon et al. [6•]. Eight patients with coronary heart disease (CHD) or equivalents were assigned to receive a single oral dose of one of the following: sucrose solution containing unformulated D-4F at $30 \mathrm{mg}, 100 \mathrm{mg}$, $300 \mathrm{mg}$, or $500 \mathrm{mg}$ or placebo (sucrose solution without D-4F) (total patients $=40$ ). An additional 10 patients received $500 \mathrm{mg}(n=8)$ or placebo $(n=2)$ with a low-fat meal. There were no significant trends in any safety parameter. D-4F was detectable in plasma at a maximal concentration of approximately $4 \mathrm{nM}$. HDL from each time point for each subject was tested for its ability to inhibit LDL-induced MCP-1 production in cultures of human aortic endothelial cells. The values obtained were normalized to 1.0 for LDL alone to obtain the HDL inflammatory index. This index significantly improved at 4 hours in participants receiving the $300-\mathrm{mg}$ dose and at 2 hours in participants receiving the 500-mg dose compared with placebo $(P<0.05)$. There were no changes in plasma lipid or lipoprotein levels. It was concluded that unformulated D-4F has low bioavailability that is improved under fasting conditions and that a single dose of D-4F is safe and well tolerated and may improve the HDL inflammatory index [6•].

\section{Conclusions}

Oxidized lipids show a remarkable binding affinity for apoA-I mimetic peptides compared with apoA-I. Oxidized lipids bind with equal affinity to peptides synthesized from D- or Lamino acids. There is no difference in peptide efficacy in reducing inflammation and lesions in a rabbit model of atherosclerosis whether the peptides are synthesized from D- or Lamino acids. The best predictors of lesion area and peptide efficacy are lipoprotein inflammatory properties and serum amyloid A levels. The apoA-I mimetic peptide $4 \mathrm{~F}$ increases the formation of pre- $\beta$ HDL, increases cholesterol efflux, and reduces lipoprotein oxidation in vitro. The peptide $4 \mathrm{~F}$ increases antioxidants and vascular repair in type 1 diabetic rats. In a mouse model of scleroderma, $4 \mathrm{~F}$ improves vasodilation, oxidative stress, myocardial inflammation, and angiogenic potential. Administration of $4 \mathrm{~F}$ reduces renal inflammation in LDL receptor-null mice fed a Western diet by reducing the kidney content of oxidized phospholipids without altering kidney retention of apoB-containing lipoproteins or plasma lipids. The peptide $4 \mathrm{~F}$ reduces arthritis in a rat model of collagen-induced arthritis and the therapeutic effect correlates with the HDL inflammatory index. Administration of 4F reduces adiposity, increases adiponectin levels, and improves insulin sensitivity in obese mice. In humans with $\mathrm{CHD}$, a single oral dose of unformulated $4 \mathrm{~F}$ producing low plasma levels improves HDL inflammatory properties. Taken together, these recent publications suggest that apoA-I mimetic peptides may be a promising new therapeutic strategy.

\section{Acknowledgments}

This work was supported in part by US Public Health Service grants HL-30568 and HL-34343. 
Dr. Anantharamaiah can be contacted at the Department of Medicine, University of Alabama at Birmingham in Birmingham, AL.

\section{References and Recommended Reading}

Papers of particular interest, published recently, have been highlighted as:

- Of importance

•• Of major importance

1. deGoma EM, deGoma RL, Rader DJ. Beyond high-density lipoprotein cholesterol levels. Evaluation high-density lipoprotein function as influenced by novel therapeutic approaches. J Am Coll Cardiol 2008;51:2199-2211. [PubMed: 18534265]

2. Datta G, Chaddha M, Hama S, et al. Effects of increasing hydrophobicity on the physical-chemical and biological properties of a class A amphipathic helical peptide. J Lipid Res 2001;42:1096-1104. [PubMed: 11441137]

3. Navab M, Anantharamaiah GM, Reddy ST, et al. Apolipoprotein A-I mimetic peptides. Arterioscler Thromb Vasc Biol 2005;25:1325-1331. [PubMed: 15831812]

4. Anantharamaiah GM, Mishra VK, Garber DW, et al. Structural requirements for antioxidative and anti-inflammatory properties of apolipoprotein A-I mimetic peptides. J Lipid Res 2007;48:19151923. [PubMed: 17570869] This is a review of the structural requirements for apoA-I mimetic peptides.

5. Navab M, Anantharamaiah GM, Reddy ST, et al. Oral D-4F causes formation of pre-beta highdensity lipoprotein and improves high-density lipoprotein-mediated cholesterol efflux and reverse cholesterol transport from macrophages in apolipoprotein E-null mice. Circulation 2004;109:r120r125.

6. Bloedon LT, Dunbar RL, Duffy D, et al. Safety, pharmacokinetics, and pharmacodynamics of oral apoA-I mimetic peptide D-4F in high-risk cardiovascular patients. J Lipid Res 2008;49:1344-1352. [PubMed: 18323573] This article is the first report of an apoA-I mimetic peptide in humans.

7. Navab M, Hama SY, Anantharamaiah GM, et al. Normal high density lipoprotein inhibits three steps in the formation of mildly oxidized low density lipoprotein: steps 2 and 3. J Lipid Res 2000;41:1495-1508. [PubMed: 10974057]

8. Van Lenten BJ, Wagner AC, Jung CL, et al. Anti-inflammatory apoA-I mimetic peptides bind oxidized lipids with much higher affinity than human apoA-I. J Lipid Res 2008;49:2302-2311. [PubMed: 18621920] This report provides the mechanism of action for the anti-inflammatory properties of apoA-I mimetic peptides

9. Van Lenten BJ, Wagner AC, Navab M, et al. Lipoprotein inflammatory properties and serum amyloid A levels but not cholesterol levels predict lesion area in cholesterol-fed rabbits. J Lipid Res 2007;48:2344-2353. [PubMed: 17693626] This article demonstrates that there is no significant difference between the $\mathrm{D}$ and $\mathrm{L}$ forms of apoA-I mimetic peptides if they are administered parenterally.

10. Troutt JS, Alborn WE, Mosior MK, et al. An apolipoprotein A-I mimetic dose-dependently increases the formation of Pre-beta1 HDL in human plasma. J Lipid Res 2008;49:581-587. [PubMed: 18056684]

11. Wool GD, Reardon CA, Getz GS. Apolipoprotein A-I mimetic peptide helix number and helix linker influence potentially anti-atherogenic properties. J Lipid Res 2008;49:1268-1283. [PubMed: 18323574]

12. Peterson SJ, Husney D, Kruger AL, et al. Long-term treatment with the apolipoprotein A1 mimetic peptide increases antioxidants and vascular repair in type I diabetic rats. J Pharmacol Exp Ther 2007;322:514-520. [PubMed: 17488882] This article demonstrates the potential use of apoA-I mimetic peptides in protecting the vasculature in diabetes.

13. Weihrauch D, Xu H, Shi Y, et al. Effects of D-4F on vasodilation, oxidative stress, angiostatin, myocardial inflammation, and angiogenic potential in tight-skin mice. Am J Physiol Heart Circ Physiol 2007;293:H1432-H1441. [PubMed: 17496220] This report demonstrates the potential use of apoA-I mimetic peptides in scleroderma. 
14. Buga GM, Frank JS, Mottino GA, et al. D-4F reduces EO6 immunoreactivity, SREBP-1c mRNA levels, and renal inflammation in LDL receptor-null mice fed a Western diet. J Lipid Res 2008;49:192-205. [PubMed: 17925450] This article demonstrates that apoA-I mimetic peptides can remove proinflammatory oxidized lipids and reduce inflammation without changing the tissue retention of apoB-containing proteins and without changing plasma lipid levels.

15. Charles-Schoeman C, Banquerigo ML, Hama S, et al. Treatment with an apolipoprotein A-1 mimetic peptide in combination with pravastatin inhibits collagen-induced arthritis. Clin Immunol 2008;127:234-244. [PubMed: 18337176]

16. Peterson SJ, Drummond G, Kim DH, et al. L-4F treatment reduces adiposity, increases adiponectin levels, and improves insulin sensitivity in obese mice. J Lipid Res 2008;49:1658-1669. [PubMed: 18426778] This article demonstrates the potential utility of apoA-I mimetic peptides in reducing adiposity, improving insulin sensitivity, and increasing adiponectin levels. 